\title{
14-3-3 proteins in apoptosis
}

M. Rosenquist
Received August 5, 2002

Accepted November 7, 2002

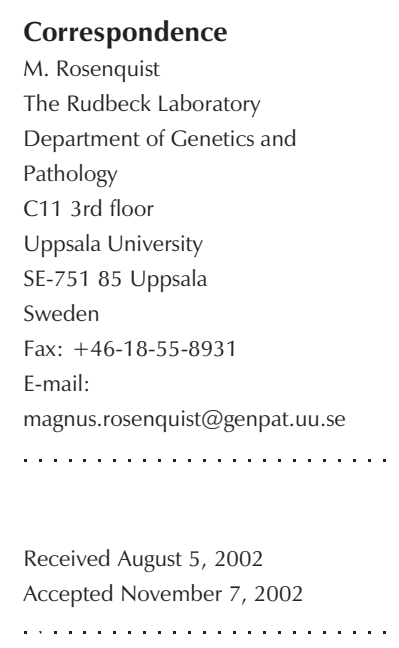

Correspondence

Department of Genetics and

$\ldots \ldots \ldots \ldots \ldots \ldots \ldots$
The Rudbeck Laboratory, Department of Genetics and Pathology, Uppsala University, Uppsala, Sweden

Abstract

The once obscure members of the 14-3-3 protein family play significant roles in the determination of cell fate. By inhibiting the proapoptotic BAD (Bcl-2-antagonist of cell death) and the transcription factor FKHRL-1, 14-3-3 displays important anti-apoptotic characteristics. To date, five points of interaction of 14-3-3 with the apoptotic machinery have been identified. How these interactions are regulated still remains a mystery.

\section{Brief background of 14-3-3}

More than three decades ago, Moore and Perez (1) fractionated soluble cow brain proteins using two-dimensional DEAE-cellulose chromatography and starch-gel electrophoresis. One of the gel spots was designated 143-3, and 14-3-3 was later shown to be an abundant protein comprising $1 \%$ of the total amount of soluble brain protein (2). Since then, 14-3-3 proteins have kept their enigmatic name as their promiscuous binding habits have implicated them in an array of cellular mechanisms which initially seemed to have no common denominator.

To date, 14-3-3s have been found in all organisms and cell types examined except for members of the Prokaryote kingdom, where intriguingly not even a putative ancestor can be found. The completion of the sequencing of the Homo sapiens and the Arabidopsis thaliana genomes has enabled the determination of the exact number of 143-3 genes in these species. In $H$. sapiens seven isoforms have been identified (14-3-3 $\beta, \gamma, \varepsilon, \eta, \sigma, \tau / \theta$, and $\zeta)$. In A. thaliana, the number of 14-3-3 genes is 15 . However, the number of expressed 14-3-3 genes in $A$. tha- liana experimentally confirmed is, to date, only 12 (GF14 $\varepsilon, 1, \kappa, \lambda, \mu, v, \mathrm{o}, \mathrm{v}, \phi, \chi, \psi$, and $\omega$ ) (3). The reasons for the presence of so many isoforms have long been debated. One reason is tissue-specific expression. Subcellular compartmentalization is another proposed theory (4), which is thwarted by the fact that yeast, with the same organellar complexity as mammals, still only have two isoforms. The consensus is that unicellular organisms have few 14-3-3 isoforms, whilst multicellular organisms have several (5).

The potential roles of 14-3-3 in signal transduction and the association with oncogene products, such as the polyoma middle T-antigen and the protein kinases Raf-1, Bcr, and Bcr-Abl, attracted a lot of attention to the 14-3-3 protein family (reviewed in Refs. 6,7). During the last couple of years, 14-3-3 proteins have been shown to be key regulators of a large number of processes such as mitosis and apoptosis in animals, and primary metabolism in plants. There are reports in the literature demonstrating interaction between 14-3-3s and an astonishing 104 different enzymes/proteins. Still, the 14-3-3 protein family remains quite neglected and unknown in the science community. 


\section{4-3-3 functions}

14-3-3 proteins function as dimers with each monomer able to bind a target. Actions can be divided into four categories: inhibition, activation, structural stabilization, or translocation. Translocation may involve the intrinsic nuclear export signal in 14-3-3 (4) and/or obstruction of a nuclear localization signal (8). Until recently, the consensus about their action was that they bind to a phosphoserine within defined target motifs. Screening a phosphopeptide library using surface plasmon resonance revealed two consensus 14-3-3 binding motifs, RSXpSXP and RXY/ FXpSXP (9). Preference for having certain amino acids in certain positions in these consensus motifs led to the determination of two optimal motifs (4), which are present in most interactants. When studying possible 14-3-3 isoform-specific binding to targets harboring any of the optimal motifs, little to no difference was observed (4). However, when more deviating binding motifs were used, substantial differences were found $(5,10)$. In light of the fact that several 14-3-3 isoforms are always present within a cell, and that specific isoforms have been identified in specific interactions involving optimal motifs (11), the question of how 14-3-3 binding is regulated arises. Phosphorylation of the target protein in the 14-3-3 binding motif, being a prerequisite for binding, is an apparent regulation. If they are promiscuous in their binding to the consensus motifs in vitro, what keeps specific 14-3-3 isoforms from binding to other interactants? In the case of mammalian 14-3-3 $\zeta$ binding to Raf1 , phosphorylation of $\zeta$ itself was shown to drastically decrease binding, suggesting that phosphorylation of 14-3-3 is also a form of regulation (12). Three additional mammalian 14-3-3 isoforms have been shown to be phosphorylated in vivo (14-3-3 $\beta, \gamma$, and $\eta$ ), whilst the remaining isoforms lack the phosphorylatable residue involved in the $\zeta$ example (reviewed in Ref. 5). The phospho- rylated forms of $14-3-3 \beta$ and $\zeta$ are commonly referred to as 14-3-3 $\alpha$ and $\delta$, respectively.

There are several examples of interactants with motifs very different from the consensus motifs, e.g., the plant plasma membrane $\mathrm{H}^{+}$ATPase with a phosphothreonine, and exoenzyme S, which has no phosphorylated residue in its binding motif(reviewed in Ref. 5). These observations suggest that a vast number of binding partners remain to be identified.

\section{Apoptosis}

The phenomenon of programmed cell death (PCD) in normal development of vertebrates and invertebrates, commonly referred to as apoptosis, was first observed in the 1950's (reviewed in Ref. 13). Apoptosis is a highly controlled type of cell death that plays a critical role in embryonic development, deletion of autoreactive immune cells, and adult tissue homeostasis (reviewed in Ref. 14). There is increasing evidence that malfunction in the apoptotic program is the underlying cause of a series of diseases. The fundamental cellular mechanism behind apoptosis can best be described as a balance between anti-apoptotic and pro-apoptotic factors, which may be shifted by extracellular signals. 14-3-3 proteins play an important suppressing role in several apoptotic pathways in animals (Figure 1). In plants, PCD is not nearly as well defined as apoptosis is in animals, and involvement of 14-3-3 proteins has yet to be shown.

In plants, 14-3-3 proteins mainly regulate primary metabolism in contrast to animals, where they function as regulators of signal transduction, synthesis of signal substances etc. The phenomenon of PCD in plants occurs histologically in a totally different manner compared to animal cells. There is no blebbing or subsequent phagocytosis, which means that PCD should not be referred to as apoptosis in plants. Furthermore, in plants, 
14-3-3 involvement in the process of PCD may be very different, as there are no homologous components of the apoptosis pathways in animals. However, an increasing amount of circumstantial evidence suggests that PCD occurs by similar pathways across the kingdoms, DNA laddering being the strongest evidence (15).

\section{4-3-3 and apoptotic pathways}

Apoptosis can be initiated by ligand binding to members of the family of membrane bound tumor necrosis factor (TNF) receptors, e.g., TNF-R1 and CD95 (Fas) (16). The core of the subsequently activated intracellular machinery in this case is formed by a

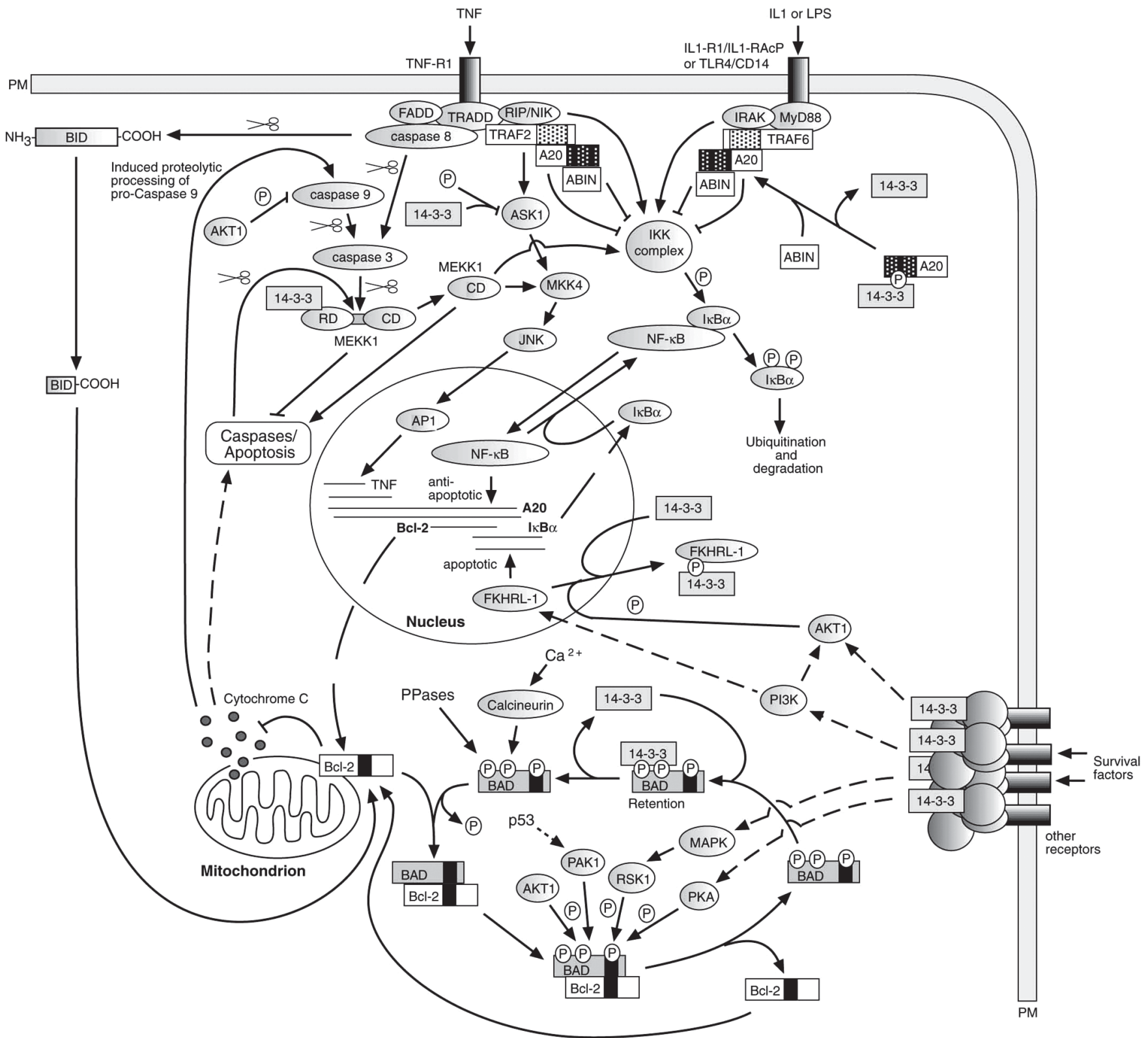

Figure 1. The complex apoptotic machinery. To date, five known 14-3-3 target proteins have been identified in the different pathways. Phosphorylation events are indicated by circled $\mathrm{P}$, and proteolytic processing is indicated by scissors. 
set of proteases, named caspases. Activation of TNF-R1 leads to recruitment of the TNF receptor-associated death domain (TRADD), which subsequently recruits the Fas-associated death domain and caspase-8 (reviewed in Ref. 17). Caspase-8 activates caspase-3, directly or via caspase-9. Caspase- 3 removes the N-terminal regulatory domain of a mitogen-activated protein kinase (MAPK)/extracellular signal-regulated kinase kinase (MEKK1) from the catalytic domain, which ultimately results in apoptosis. 14-3-3 binds to the regulatory domain of MEKK1 (18), but neither the specific 14-3-3 isoforms involved nor the purpose of this binding have been determined. This interaction may constitute a link between the MAPK signal transduction pathway and apoptosis.

TNF binding to TNF-R1 also activates the recruitment of other TNF receptor-associated factors to TRADD, which via signal transduction ultimately activates the antiapoptotic transcription factor nuclear factor $\kappa \mathrm{B}(\mathrm{NF}-\kappa \mathrm{B})$. The apoptosis-suppressing activity of NF- $\kappa B$ includes regulation of expression of the zinc finger protein A20 and anti-apoptotic genes (19). A recent study has confirmed earlier observations that 14-3-3 isoforms $(B, \varepsilon, \eta$, and $\zeta)$ bind $A 20$, but has shown that the binding is not a prerequisite for the inhibitory effect A20 exerts on the TNF pathway (20). In addition, the binding of 14-3-3 to A20 has been shown to mediate interaction with the c-Raf kinase (21), implicating a role for A20 in signal transduction and providing another 14-3-3-involving link between signal transduction and apoptosis.

Intriguingly, 14-3-3 isoforms $\eta$ and $\zeta$ bind to the zinc finger domain of A20, which must result in blocking other proteins from interacting with this domain (22), e.g., the A20-binding inhibitor of NF- $\mathrm{BB}, \mathrm{ABIN}$ (murine HIV Nef-associated factor 1 homolog) (19). Moreover, the practical effect of A20 on apoptosis seems to be to simply inhibit

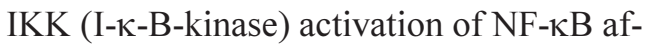

ter apoptosis has been prevented. With its nuclear localization signal, exposed NF- $\mathrm{kB}$ translocates to the nucleus, where it binds to $\kappa B$ sites in the promoter region of NF- $\kappa B$ responsive genes. In addition, interleukin-1 and lipopolysaccharides have been found to up-regulate A20 in endothelial cells, via a pathway similar to the TNF pathway, converging at the IKK complex (reviewed in Ref. 19).

Overexpression of A20 prevents NF- $\kappa B-$ dependent expression of $\mathrm{I} \kappa \mathrm{B} \alpha$, demonstrating what may be interpreted as an apoptotic action of A20 (19), suggesting that A20 is part of a negative feedback regulation NF$\kappa B$ exerts on itself. At the same time, NF- $\kappa B$ also activates expression of genes such as the anti-apoptotic Bcl-2 (reviewed in Ref. 17), thus possibly transcribing I $\mathrm{I} B \alpha$ and A20 to switch itself off after a sufficient amount of anti-apoptotic proteins have been produced. Intriguingly, studies have shown that overexpression of A20 suppresses apoptosis in some cell lines whilst induction is observed in others (reviewed in Ref. 19). The amount of other proteins involved in apoptosis most likely determines the fate of these cells, which then might simply be affected by elevated A20 levels.

\section{4-3-3 and the Bcl-2 family}

Another important regulatory protein family with regard to apoptosis is the Bcl-2 family. Its members include pro-apoptotic proteins (e.g., BAD, BID, BAX, Bak, Bcl$\mathrm{X}_{\mathrm{S}}$ ) as well as anti-apoptotic proteins (e.g., Bcl-2, Bcl- $\left.X_{\mathrm{L}}, \mathrm{Bfl}-1 / \mathrm{A} 1, \mathrm{Mcl}-1\right)$. The antiapoptotic actions consist of anchoring to the outer membrane of mitochondria, controlling ion flux and cytochrome c release.

Of all the Bcl-2 family proteins, BAD (Bcl-2-antagonist of cell death) is the most extensively studied and the only member for which 14-3-3 binding has been demonstrated. Three phosphorylation sites have been identified on BAD: Ser112, Ser136, and Ser155, which are phosphorylated by a variety of 
kinases (23). The phosphorylation of Ser155, which is located in the BH3-interacting domain, disrupts the binding of $\mathrm{Bcl}-2$, resulting in return to the mitochondrial membrane and suppression of apoptosis. Upon dephosphorylation, 14-3-3 dissociates and BAD may again bind and translocate Bcl-2 (24-26). In a heterologous experiment, all tested 14-3-3 isoforms were able to bind BAD (27). Muslin and Xing (8) proposed a model for the 143-3/BAD interaction that involves obstruction of the Bcl-2-binding $\mathrm{BH} 3$ domain. This proposed model for molecular interference, i.e., the obstruction of another functional domain in a 14-3-3 target protein, applies to other 14-3-3 interactions as well.

\section{4-3-3 and transcription factors}

Activation of transcription factors, and subsequent gene expression, is an apparently important process in maintaining the balance between agonists and antagonists of apoptosis. FKHRL-1 (Forkhead transcription factor 1) reportedly transcribes apoptosis-promoting genes, and its mechanism of regulation resembles that of $\mathrm{NF}-\mathrm{\kappa B}$. AKT (a.k.a. PKB, protein kinase B) phosphorylates DAF-16, a Caenorhabditis elegans FKHRL-1 homolog, on Thr54, Ser240/242, and Ser314. 14-3-3 subsequently binds to the pThr54 and pSer314 sites, and translocates the complex to the cytoplasm (28). No apparent nuclear localization signal has been found on FKHRL-1, and the nuclear export signal may be supplied by 14-3-3. Intriguingly, a report by Cahill et al. (28) showed that phosphatidylinositol-3-kinase may circumvent the AKT-mediated inhibition of DAF-16 in cells expressing DAF-16 mutated at the AKT/14-3-3 sites. This advocates the existence of a second mechanism of regulation independent of AKT and 14-3-3.

\section{4-3-3 and apoptosis-related kinases}

The kinase AKT exerts its anti-apoptotic role at several points in the apoptotic machinery (Figure 1). Besides phosphorylating BAD and FKHRL-1, AKT also inactivates caspase- 9 by phosphorylation on the Ser196 residue (29). In contrast to FKHRL-1 and $\mathrm{BAD}$, no subsequent interaction between 14-3-3 and caspase-9 has yet been observed. Apoptosis signal-regulating kinase 1 (ASK1) associates with TRAF2 (TNF-R-associating factor 2), and mediates activation of the transcription factor AP1 via the Jun N-terminal kinase pathway, resulting in TNF expression (30). Phosphorylation and 14-3-3 binding to ASK1 occurs at the RSIS ${ }_{967} \mathrm{LP}$ motif, and results in inhibition of the pathway (30).

\section{Conclusions}

Since 14-3-3 proteins are involved in a great number of interactions involving the optimal motifs, which possibly are indifferent to isoform specificity, knocking or overexpressing specific 14-3-3 genes or 14-3-3 target genes will most likely have an adverse effect on the other 14-3-3-regulated cell processes. This effect, e.g., shift in the equilibrium between agonists and antagonists of apoptosis, which is essential to maintain, may also occur if the amount of one binding partner is altered, e.g., A20. In such cases, endogenous 14-3-3 will be bound by an excess amount of introduced target protein. Thus, the effects observed in heterologous experiments involving expression of metazoan anti-apoptotic proteins in plants (15) may simply be the result of disturbed cellular equilibrium. Similarly, contradictory results may be obtained when the same gene is overexpressed in different cell lines.

In approximately 30 out of the total of 104 complexes involving 14-3-3s reported, specific isoforms have been identified. Only the 14-3-3 $\sigma$ isoform seems to be involved in all interactions identified in cell cycle regulation (11). Could a single isoform be involved in all interactions identified in apoptosis too? If so, which isoform is it, and what 
makes that isoform pathway specific in vivo if 14-3-3 proteins are promiscuous in vitro? In order to answer this question, the isoforms participating in different interactions in vivo must first be identified. Only then can we begin to elucidate the mechanism behind 14-3-3 interactions in apoptosis.

\section{References}

1. Moore BW \& Perez VJ (1967). Specific acid proteins in the nervous system. In: Carlson FD (Editor), Physiological and Biochemical Aspects of Nervous Integration. Prentice Hall/Woods Hole, Woods Hole, MA, USA. 343-359.

2. Boston PF, Jackson P \& Thompson RJ (1982). Human 14-3-3 protein: radioimmunoassay, tissue distribution, and cerebrospinal fluid levels in patients with neurological disorders. Journal of Neurochemistry, 38: 1475-1482.

3. Rosenquist M, Alsterfjord M, Larsson C \& Sommarin M (2001). Data mining the Arabidopsis genome reveals fifteen 14-3-3 genes. Expression is demonstrated for two out of five novel genes. Plant Physiology, 127: 142-149.

4. Rittinger K, Budman J, Xu J, Volinia S, Cantley LC, Smerdon SJ, Gamblin SJ \& Yaffe MB (1999). Structural analysis of 14-3-3 phosphopeptide complexes identifies a dual role for the nuclear export signal of 14-3-3 in ligand binding. Molecular Cell, 4: 153-166.

5. Rosenquist M, Sehnke P, Ferl RJ, Sommarin M \& Larsson C (2000). Evolution of the 14-3-3 protein family: does the large number of isoforms in multicellular organisms reflect functional specificity? Journal of Molecular Evolution, 51: 446-458.

6. Ferl RJ (1996). 14-3-3 proteins and signal transduction. Annual Review of Plant Physiology and Plant Molecular Biology, 47: 49-73.

7. Meili R, Cron P, Hemmings BA \& Ballmer-Hofer K (1998). Protein kinase B/AKT is activated by polyomavirus middle-T antigen via a phosphatidylinositol 3-kinase-dependent mechanism. Oncogene, 16: 903-907.

8. Muslin AJ \& Xing H (2000). 14-3-3 proteins: regulation of subcellular localization by molecular interference. Cellular Signalling, 12: 703709.

9. Yaffe MB, Rittinger K, Volinia S, Caron PR, Aitken A, Leffers H, Gamblin SJ, Smerdon SJ \& Cantley LC (1997). The structural basis for 14-3-3 phosphopeptide binding specificity. Cell, 91: 961-971.

10. Athwal GS, Lombardo CR, Huber JL, Masters SC, Fu H \& Huber SC (2000). Modulation of 14-3-3 protein interactions with target polypeptides by physical and metabolic effectors. Plant and Cell Physiology, 41: 523-533.

11. Laronga $\mathrm{C}$, Yang HF, Neal C \& Lee MH (2000). Association of the cyclin-dependent kinases and 14-3-3 sigma negatively regulates cell cycle progression. Journal of Biological Chemistry, 275: 23106-23112.

12. Dubois T, Rommel C, Howell S, Steinhussen U, Soneji Y, Morrice N, Moelling K \& Aitken A (1997). 14-3-3 is phosphorylated by casein kinase I on residue 233. Phosphorylation at this site in vivo regulates Raf/14-3-3 interaction. Journal of Biological Chemistry, 272: 2888228888.

13. Gerschenson LE \& Rotello RJ (1992). Apoptosis: A different type of cell death. FASEB Journal, 6: 2450-2455.

14. Schuchmann M \& Galle PR (2001). Apoptosis in liver disease. European Journal of Gastroenterology and Hepatology, 13: 785-790.

15. Dickman MB, Park YK, Oltersdorf T, Li W, Clemente T \& French R (2001). Abrogation of disease development in plants expressing animal antiapoptotic genes. Proceedings of the National Academy of Sciences, USA, 98: 6957-6962.

16. Wallach D, Varfolomeev EE, Malinin NL, Goltsev YV, Kovalenko AV
\& Boldin MP (1999). Tumor necrosis factor receptor and Fas signaling mechanisms. Annual Review of Immunology, 17: 331-367.

17. Aggarwal BB (2000). Apoptosis and nuclear factor-кB: a tale of association and dissociation. Biochemical Pharmacology, 60: 1033-1039.

18. Fanger GR, Widmann C, Porter AC, Sather S, Johnson GL \& Vaillancourt RR (1998). 14-3-3 proteins interact with specific MEK kinases. Journal of Biological Chemistry, 273: 3476-3483.

19. Beyaert R, Heyninck K \& Van Huffel S (2000). A20 and A20-binding proteins as cellular inhibitors of nuclear factor-kB-dependent gene expression and apoptosis. Biochemical Pharmacology, 60: 11431151.

20. Lademann U, Kallunki T \& Jaattela M (2001). A20 zinc finger protein inhibits TNF-induced apoptosis and stress response early in the signaling cascades and independently of binding to TRAF2 or 14-3-3 proteins. Cell Death and Differentiation, 8: 265-272.

21. Vincenz C \& Dixit VM (1996). 14-3-3 proteins associate with A20 in an isoform-specific manner and function both as chaperone and adapter molecules. Journal of Biological Chemistry, 271: 2002920034.

22. De Valck D, Heyninck K, Van Criekinge W, Vandenabeele P, Fiers W \& Beyaert R (1997). A20 inhibits NF-kB activation independently of binding to 14-3-3 proteins. Biochemical and Biophysical Research Communications, 238: 590-594.

23. Datta SR, Katsov A, Hu L, Petros A, Fesik SW, Yaffe MB \& Greenberg ME (2000). 14-3-3 proteins and survival kinases cooperate to inactivate BAD by BH3 domain phosphorylation. Molecular Cell, 6: 41-51.

24. Springer JE, Azbill RD, Nottingham SA \& Kennedy SE (2000). Calcineurin-mediated BAD dephosphorylation activates the caspase3 apoptotic cascade in traumatic spinal cord injury. Journal of Neuroscience, 20: 7246-7251.

25. Ayllon V, Cayla X, Garcia A, Roncal F, Fernandez R, Albar JP, Martinez C \& Rebollo A (2001). Bcl-2 targets protein phosphatase $1 \alpha$ to BAD. Journal of Immunology, 166: 7345-7352.

26. Chiang CW, Harris G, Ellig C, Masters SC, Subramanian R, Shenolikar S, Wadzinski BE \& Yang E (2001). Protein phosphatase 2A activates the proapoptotic function of BAD in interleukin-3-dependent lymphoid cells by a mechanism requiring 14-3-3 dissociation. Blood, 97: 1289-1297.

27. Subramanian RR, Masters SC, Zhang H \& Fu H (2001). Functional conservation of 14-3-3 isoforms in inhibiting Bad-induced apoptosis. Experimental Cell Research, 271: 142-151.

28. Cahill CM, Tzivion G, Nasrin N, Ogg S, Dore J, Ruvkun G \& AlexanderBridges M (2001). Phosphatidylinositol 3-kinase signaling inhibits DAF-16 DNA binding and function via 14-3-3-dependent and 14-3-3independent pathways. Journal of Biological Chemistry, 276: 1340213410.

29. Cardone MH, Roy N, Stennicke HR, Salvesen GS, Franke TF, Stanbridge E, Frisch S \& Reed JC (1998). Regulation of cell death protease caspase-9 by phosphorylation. Science, 282: 1318-1321.

30. Zhang L, Chen J \& Fu H (1999). Suppression of apoptosis signalregulating kinase 1-induced cell death by 14-3-3 proteins. Proceedings of the National Academy of Sciences, USA, 96: 8511-8515. 